Sādhanā Vol. 40, Part 2, April 2015, pp. 411-423. (C) Indian Academy of Sciences

\title{
A generalized cellular automata approach to modeling first order enzyme kinetics
}

\author{
ABHISHEK DUTTA ${ }^{1, *, \dagger}$, SAURAJYOTI KAR ${ }^{2}$, ADVAIT APTE $^{3}$, \\ INGMAR NOPENS $^{1}$ and DENIS CONSTALES ${ }^{4}$
}

\author{
${ }^{1}$ BIOMATH, Department of Mathematical Modelling, Statistics and Bioinformatics, \\ Ghent University, Coupure Links 653, 9000 Ghent, Belgium \\ ${ }^{2}$ Department of Biotechnology, National Institute of Technology, \\ Durgapur 713 209, India \\ ${ }^{3}$ Biological Systems Engineering Department, Virginia Tech, \\ Blacksburg, VA 24061, USA \\ ${ }^{4}$ Department of Mathematical Analysis, Ghent University, Galglaan 2, \\ B-9000 Ghent, Belgium \\ ${ }^{\dagger}$ Present address: Departement Materiaalkunde, KU Leuven, Kasteelpark Arenberg \\ 44 bus 2450, B-3001 Heverlee-Leuven, Belgium \\ e-mail: abhishek.dutta@kuleuven.be
}

MS received 14 December 2012; revised 7 July 2014; accepted 22 December 2014

\begin{abstract}
Biochemical processes occur through intermediate steps which are associated with the formation of reaction complexes. These enzyme-catalyzed biochemical reactions are inhibited in a number of ways such as inhibitors competing for the binding site directly, inhibitors deforming the allosteric site or inhibitors changing the structure of active substrate. Using an in silico approach, the concentration of various reaction agents can be monitored at every single time step, which are otherwise difficult to analyze experimentally. Cell-based models with discrete state variables, such as Cellular Automata (CA) provide an understanding of the organizational principles of interacting cellular systems to link the individual cell (microscopic) dynamics with a particular collective (macroscopic) phenomenon. In this study, a CA model representing a first order enzyme kinetics with inhibitor activity is formulated. The framework of enzyme reaction rules described in this study is probabilistic. An extended von Neumann neighborhood with periodic boundary condition is implemented on a twodimensional (2D) lattice framework. The effect of lattice-size variation is studied followed by a sensitivity analysis of the model output to the probabilistic parameters which represent various kinetic reaction constants in the enzyme kinetic model. This provides a deeper insight into the sensitivity of the CA model to these parameters. It is observed that cellular automata can capture the essential features of a discrete real system, consisting of space, time and state, structured with simple local rules without making complex implementations but resulting in complex but explainable patterns.
\end{abstract}

*For correspondence 
Keywords. Cellular automata; enzyme kinetics; extended von-Neumann neighborhood.

\section{Introduction}

Over the past two decades, there has been a significant growth in the use of computer-generated models to study dynamic phenomena in biochemical systems (Kier et al 2005). The need to include greater details about biochemical reaction systems has led to ever-increasing demand for computational resources. Because of the complexity of such systems, it is imperative to generate huge amounts of simulation data to describe a system objectively and predict its behavior. Cellular Automata (CA) is a discrete representation of natural systems (Wolfram 1984). It deals with cells (nodes or sites) arranged in a lattice that are regular, discrete and having a set of states i.e. the features of the cells at any given time and a set of rules that affect the states (Deutsch \& Dormann 2005). It represents dynamic system discretely in space, time, and state (Apte et al 2008) and can be defined as a class of spatially and temporally dynamic, stochastic systems based on local interactions (De Levie 2000; Goutsias 2007). The representing grid can be one dimensional or of multiple dimensions. Each cell or node of the grid can represent one or multiple agents, depending on model considerations. The rules are applied to the agents represented in the grid on a neighborhood basis. Each cell is governed by local rules (Von Neumann 1966). By local, it is meant that a cell will be modified only due to its neighboring cells and not due to the overall lattice. The rules are applied to the cell under consideration, and the necessary changes in state take place. For any other cell, the interactions are completely independent of the previous rules applied on its neighboring cells. A more accurate representation of processes, dependent on the behavior of a small number of molecules of relevance in both chemistry and biochemistry, can be achieved through stochastic modeling (Seybold et al 1997). In fact, the intrinsic nature of any chemical reaction is stochastic as it has been pointed out by Van der Weeën et al (2011). A local transition rule specifies a time- and space-independent probability distribution of next states for each possible neighborhood configuration. The lattice can be one-, two- or three-dimensional. Optimization techniques using Genetic Algorithm could be considered to further optimize the parameters of the CA model (Kar et al 2014). A two-dimensional (2D) lattice has a particular boundary condition which defines the limits of the working space (Martin et al 1984). There are three boundary conditions namely periodic, reflecting, and fixed. Periodic boundaries are obtained by periodically extending the lattice, reflective boundary conditions are obtained by reflecting the lattice at the boundary while fixed boundary conditions are obtained by prescribing a fixed value for the lattice cells on the boundary and the agent is lost from the system past the edge. The local rules applied microscopically describe global characteristic and provide a macroscopic property of the system. The rules can be applied in a continuous manner or in a discrete manner. Principally, there are three types of neighborhood: von-Neumann, Moore and Radial. In a von-Neumann neighborhood (figure 1a) one node in each axial direction of the central lattice is considered. For a Moore neighborhood (figure 1b), in addition to von-Neumann, one node in each of the diagonal directions is also considered while in a radial neighborhood, the nodes that fall inside a certain radial limit around the central node are considered (figure 1c). In this study, a CA model with extended von Neumann neighborhood with two nodes in each of the axial four directions (figure 1d) is implemented to describe enzyme kinetics based on Michaelis-Menten equation as in Seybold et al (1997), Kier et al (1999) and Weimar (2002). The cellular automata enzyme kinetic model, presented in this study, is based on an understanding of the results obtained with our previous study (Kar et al 2010). In the previous formulation, the various orders of rule application were analyzed and a bias was detected 


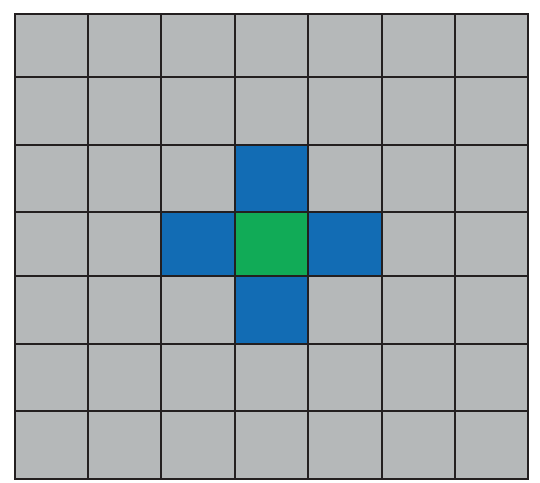

(a) von-Neumann neighborhood

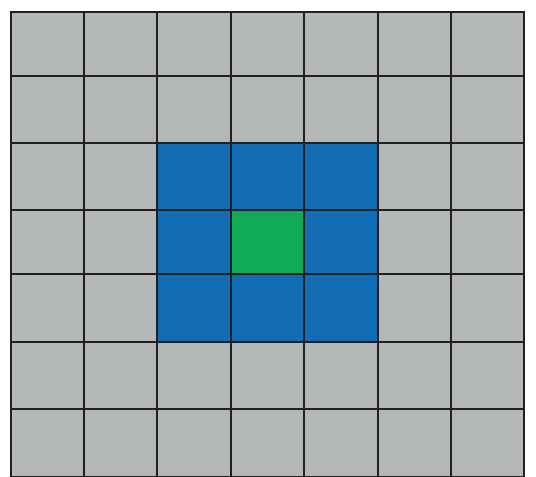

(b) Moore neighborhood

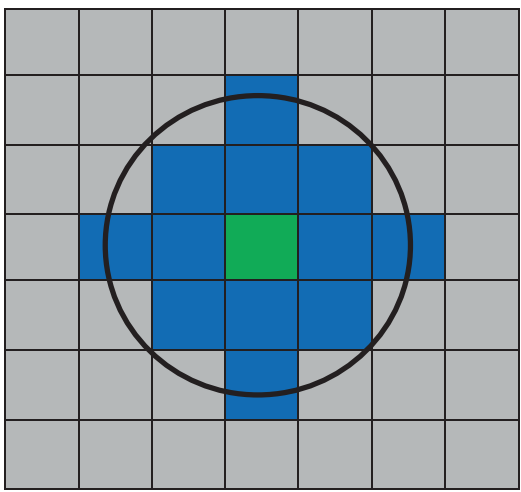

(c) Radial neighborhood

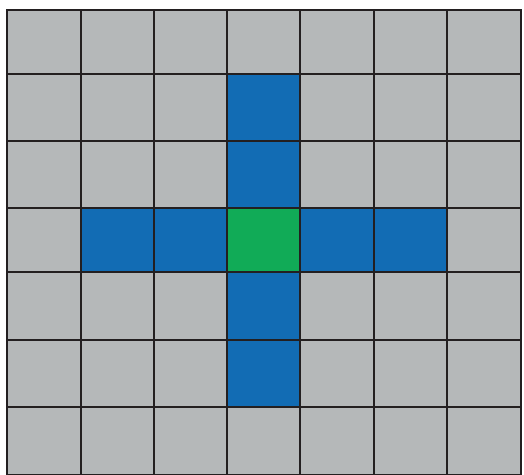

(d) Extended von-Neumann neighborhood

Figure 1. (a) von-Neumann neighborhood. (b) Moore neighborhood. (c) Radial neighborhood. (d) Extended von-Neumann neighborhood.

in all the cases studied thereby indicating that a random ordered rule application is probably the best way to simulate natural systems. The present study implements a probabilistic CA model, based on a 2D lattice, in which the interactions between the reacting systems are random in nature. Although the previous study focused on finding a CA model for first order inhibition kinetics, the present study considers a more generalized aspect of enzymatic reaction with flexibility of reaction interactions between all possible two-agent combinations. Furthermore, in the previous formulation each of the probabilistic reaction events was considered to be independent but not random; while with the current formulation, it is considered to be independent and random making it more general and realistic. The explicit novelty of this study lies in the rigorous simulation studies that are performed to understand the effects of variation of the model parameters on simulation results.

\section{Mathematical modeling}

\subsection{Model representation}

The choice of representation of the physical system in silico is the most critical step for any modeling approach. A cellular automata model on a 2D square lattice is considered where each 
of the lattice nodes can be occupied by only one agent or can remain void. To eliminate the implementation of separate boundary conditions and to make the reaction surface a continuous one, periodic boundary has been considered causing the lattice to behave as a toroid. The rules are applied on an extended von-Neumann neighborhood. The chemical kinetic agents considered in this study are Substrate (S), Product (P), Water (W), Enzyme (E) and Inhibitor (I). The model consists of the following kinetic equations:

$$
\begin{aligned}
& \mathrm{E}+\mathrm{S} \underset{\mathrm{K}-1}{\stackrel{\mathrm{K}_{1}}{\rightleftarrows}} \mathrm{ES} \underset{\mathrm{K}_{-2}}{\stackrel{\mathrm{K}_{2}}{\rightleftarrows}} \mathrm{EP} \underset{\mathrm{K}_{-3}}{\stackrel{\mathrm{K}_{3}}{\rightleftarrows}} \mathrm{E}+\mathrm{P} \\
& \mathrm{E}+\mathrm{I} \underset{\mathrm{K}_{-4}}{\stackrel{\mathrm{K}_{4}}{\rightleftarrows}} \mathrm{EI} \\
& \mathrm{W}+\mathrm{S} \underset{\mathrm{K}}{\stackrel{\mathrm{K}_{5}}{\rightleftarrows}} \mathrm{WS} \\
& \mathrm{W}+\mathrm{P} \underset{\mathrm{K}_{-6}}{\stackrel{\mathrm{K}_{6}}{\rightleftarrows}} \mathrm{WP}
\end{aligned}
$$

where the $\mathrm{k}_{\mathrm{i}}$ values represent the reaction constants. Positive subscripts represent reaction rate constants of forward reactions while negative subscripts indicate reaction rate constants for reverse reactions. Note that water is explicitly considered in the model as it has been used in most of the earlier cellular automata studies related to enzyme kinetics (for example, Seybold et al 1997; Kier et al 1999 and Apte et al 2008).

\subsection{Model rules}

The stochastic CA model presented in this study has probabilistic rules to control the state dynamics. It is based on a one-to-one agent interaction. The rules are based on four probabilities input into the system namely Joining probability $\left(\mathrm{P}_{\mathrm{J}}\right)$, Breaking probability $\left(\mathrm{P}_{\mathrm{B}}\right)$, Transition probability $\left(\mathrm{P}_{\mathrm{R}}\right)$ and Free Movement probability $\left(\mathrm{P}_{\mathrm{m}}\right)$. When two agents are adjacent to each other, they may participate in joining. As per the reaction structure (see Eq. 1), each agent can only be joined to another agent at any time. The joining probability $\mathrm{P}_{\mathrm{J}}$ is the likelihood of an agent to join another agent. Two adjacent agents, if already joined, can participate in breaking. The breaking probability $\mathrm{P}_{\mathrm{B}}$ is the measure of likelihood an agent can break from the other. If two agents are joined, and the complex can transform to another state, the transition probability rule is applied. The transition probability $\mathrm{P}_{\mathrm{R}}$ represents the likelihood of the reaction to occur. But if an agent is un-joined and the adjacent node is void, it can participate in free movement with empty adjacent cell available within the neighborhood. The free movement probability $\mathrm{P}_{\mathrm{m}}$ is a measure of Brownian motion in the reaction system. The joining, breaking and the transition rules have kinetic analogue. The equation analogue of the cellular automata model is indicated in table 1 . The model follows asynchronous rule application, meaning no two events (agent dynamics) occur simultaneously however small the time scale is considered and the rules are applied on a one-to-one basis in a randomized manner. With each iteration, the agents are randomly chosen for rule application and each agent gets one chance for an update of its state. The order in which the agents are scanned per iteration is random and hence, given the current state of the system, the state after the next iteration cannot be precisely predicted. The automata rules are being applied on the basis of select-scan-implement basis for each of the agents. First, the lattice neighborhood is scanned for free nodes and occupied nodes. The current agent can move to the free neighboring sites or can undergo transitions with the occupied ones. For each of the feasible movement directions the breaking probabilities, the joining probability and the 
Table 1. Representation of the model parameters, classified under the various probabilities considered in the model, and their analogous kinetic reaction constants.

\begin{tabular}{|c|c|c|c|c|c|c|}
\hline \multicolumn{2}{|c|}{$\begin{array}{c}\text { Joining } \\
\text { probability }\left(\mathrm{P}_{\mathrm{J}}\right)\end{array}$} & \multicolumn{2}{|c|}{$\begin{array}{c}\text { Breaking } \\
\text { probability }\left(\mathrm{P}_{\mathrm{B}}\right)\end{array}$} & \multicolumn{2}{|c|}{$\begin{array}{c}\text { Transition } \\
\text { probability }\left(\mathrm{P}_{\mathrm{T}}\right)\end{array}$} & \multirow{2}{*}{$\begin{array}{c}\begin{array}{c}\text { Free movement } \\
\text { probability }\left(\mathrm{P}_{\mathrm{m}}\right)\end{array} \\
\begin{array}{c}\text { Model } \\
\text { parameter }\end{array}\end{array}$} \\
\hline $\begin{array}{l}\text { Kinetic } \\
\text { constant }\end{array}$ & $\begin{array}{c}\text { Model } \\
\text { parameter }\end{array}$ & $\begin{array}{l}\text { Kinetic } \\
\text { constant }\end{array}$ & $\begin{array}{c}\text { Model } \\
\text { parameter }\end{array}$ & $\begin{array}{l}\text { Kinetic } \\
\text { constant }\end{array}$ & $\begin{array}{c}\text { Model } \\
\text { parameter }\end{array}$ & \\
\hline $\mathrm{K}_{1}$ & Join(ES) & $\mathrm{K}-1$ & Break(ES) & $\mathrm{K}_{2}$ & Transition $(\mathrm{S})$ to $(\mathrm{P})$ & Move W \\
\hline $\mathrm{K}_{3}$ & Join(EP) & $\mathrm{K}-3$ & Break(EP) & & & Move S \\
\hline $\mathrm{K}_{4}$ & Join(EI) & $\mathrm{K}-4$ & Break(EI) & $\mathrm{K}_{2}$ & Transition $(\mathrm{P})$ to $(\mathrm{S})$ & Move $\mathrm{P}$ \\
\hline $\mathrm{K}_{5}$ & Join(WS) & $\mathrm{K}-5$ & Break(WS) & & & Move E \\
\hline $\mathrm{K}_{6}$ & Join(WP) & $\mathrm{K}-6$ & Break(WP) & & & Move I \\
\hline
\end{tabular}

free movement probability are individually multiplied. Next, all the possible directional events are re-normalized if their sum is greater than one. Among the applicable events, one is randomly selected and implemented. The event might either happen or not at all. Each of the agents is similarly tested for the state dynamics. The flowchart shown in figure 2 indicates the steps involved in the scanning of a single agent on a CA lattice.

\subsection{Model parameters}

The model parameters along with their kinetic constants, classified under different probabilities, designed for an enzymatic system used in the present study are listed in table 1. Along with these values, the concentration of five different agents namely S, P, W, E and I constitutes the model parameters that describe the dynamics of the initial state. The input parameter values considered in the model are listed in table 2. Each of these parameters is varied to analyse its effect on overall performance of the model and to correlate the results with the kinetic properties of the model. Variation of each parameter at a time is performed with a step increment of 0.01 ranging from 0 to 0.99 , while the values of the other parameters are kept constant to their standard values.

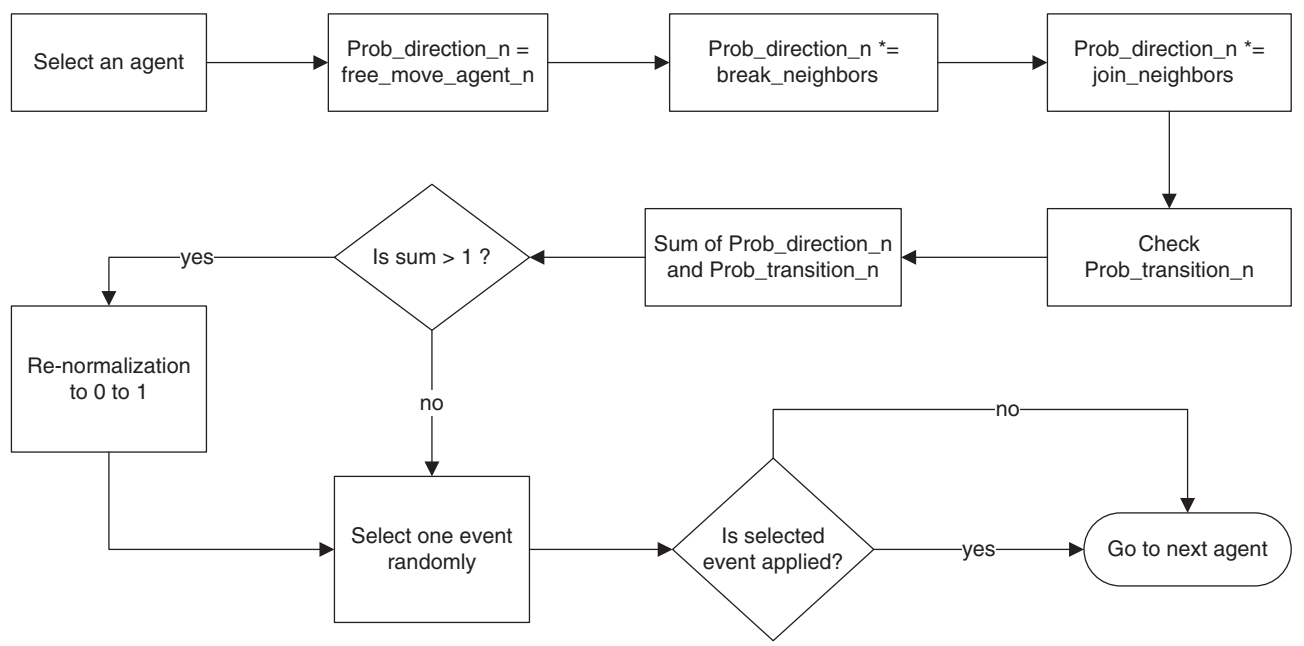

Figure 2. Flowchart of the cellular automata model for the enzymatic reaction. 
Table 2. Input parameter values considered in the model simulation.

\begin{tabular}{|c|c|c|c|c|c|c|c|c|c|}
\hline \multicolumn{2}{|c|}{$\begin{array}{c}\text { Agent } \\
\text { concentration }\end{array}$} & \multicolumn{2}{|c|}{$\begin{array}{c}\text { Joining } \\
\text { probability }\end{array}$} & \multicolumn{2}{|c|}{$\begin{array}{l}\text { Breaking } \\
\text { probability }\end{array}$} & \multicolumn{2}{|l|}{$\begin{array}{l}\text { Transition } \\
\text { probability }\end{array}$} & \multicolumn{2}{|c|}{$\begin{array}{l}\text { Free movement } \\
\text { probability }\end{array}$} \\
\hline$\overline{\text { Agent }}$ & $\%$ & Parameter & $\overline{\text { Value }}$ & Parameter & Value & Parameter & $\overline{\text { Value }}$ & Parameter & $\overline{\text { Value }}$ \\
\hline $\mathrm{W}$ & 60 & Join(ES) & 0.5 & Break(ES) & 0.5 & Transition $(\mathrm{S})$ to $(\mathrm{P})$ & 0.5 & Move W & 0.5 \\
\hline S & 4 & Join(EP) & 0.5 & Break(EP) & 0.5 & Transition $(\mathrm{P})$ to $(\mathrm{S})$ & 0.5 & Move $\mathrm{S}$ & 0.5 \\
\hline $\mathrm{P}$ & 0 & Join(EI) & 0.5 & Break(EI) & 0.5 & & & Move $\mathrm{P}$ & 0.5 \\
\hline $\mathrm{E}$ & 1 & Join(WS) & 0.5 & Break(WS) & 0.5 & & & Move E & 0.5 \\
\hline I & 1 & Join(WP) & 0.5 & $\operatorname{Break}(\mathrm{WP})$ & 0.5 & & & Move I & 0.5 \\
\hline Void & 34 & & & & & & & & \\
\hline
\end{tabular}

The results presented in this study show the variation of the product concentration after 3000 iterations of simulation run per set of input parameter values.

\section{Results and discussions}

The cellular automata model presented in this study is based on Kar et al (2010) in which various orders of rule application were analyzed and a bias was detected, indicating that a random ordered rule application is the best way to simulate biochemical systems. The reaction events are built by conditional probabilities and are completely independent of each other. Simulations of the kinetic model were performed to study the change in concentration of agents over time. The concentrations of S and P and complexes ES and EP were measured. For the first simulation case, all free movement probability parameters were fixed to 1 . The joining probabilities, breaking probabilities and the transition probabilities were fixed to 0.5 . The concentration profile is shown in figure $3 \mathrm{a}$. At the initial phase of reaction the concentration of $\mathrm{S}$ remains high, which drives the reaction in forward direction. When the concentration of $S$ equals the concentration of $\mathrm{P}$, the reaction reaches equilibrium. The concentrations of ES and EP complexes remain fairly constant throughout the reaction time. In the second simulation case, the parameter values were set so that only the interactions between $\mathrm{E}, \mathrm{S}$ and $\mathrm{P}$ were allowed (value fixed to 0.5 ). The other parameters of joining and breaking with respect to $\mathrm{W}$ and I were fixed to 0 . The free movement parameters were fixed to 1 , as in previous case. The concentration profile of different agents and complex is shown in figure $3 \mathrm{~b}$. The reaction reaches equilibrium after a number of iteration steps, but here the concentrations of $\mathrm{S}$ and $\mathrm{P}$ do not attain equality. It has been observed that for any combination of the parameter values, the model is able to reach equilibrium by 3000 iteration steps. Henceforth the analyses presented in this study comply with the number of iterations for each model run being fixed to 3000.These two simulation studies clearly show that the CA model is able to describe the general chemical kinetics accurately. A snapshot of the lattice configuration of the enzymatic reaction can be seen in figure 4. Several features of the enzymatic reaction CA code can be seen through the several rings encircled in the lattice space. From the top, enzyme-Substrate can be seen in a complex (ES). Below, the enzyme is seen that may enter into a complex with either of the neighboring substrate $(\mathrm{E}+\mathrm{S})$. Further down, once the reaction is successful the complex is seen to be broken; the product separates and is free to move randomly $(\mathrm{E}+\mathrm{P})$. At the bottom end, it is seen that enzyme is already in a complex, but may react with its neighboring substrate to form a new complex. 

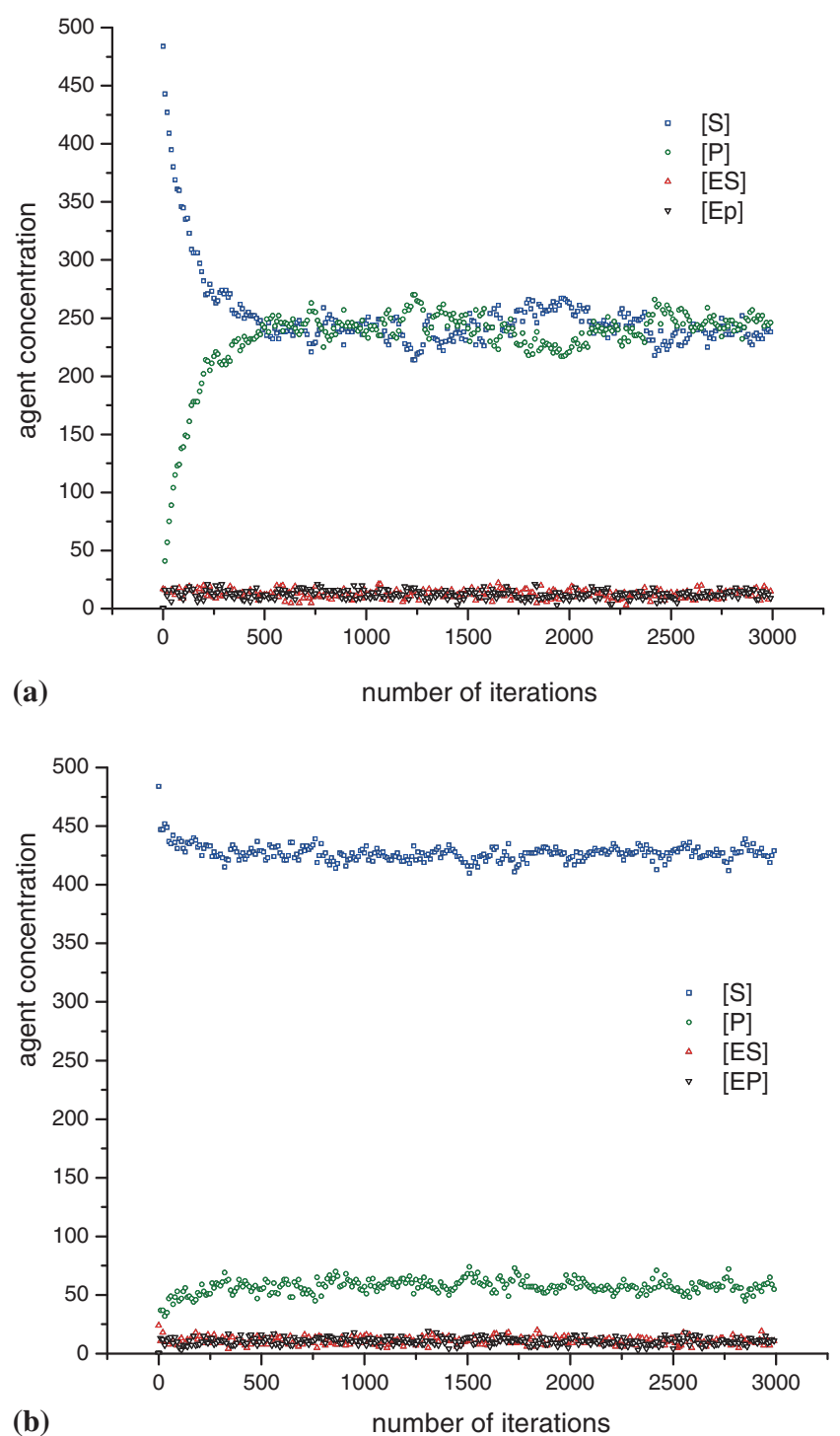

Figure 3. (a) Variation of agent concentrations (S, P, ES, EP) with iteration, considering fixed reaction parameters $(=0.5)$. (b) Variation of agent concentrations (S, P, ES, EP) with iteration considering only interactions between $\mathrm{E}, \mathrm{S}$, and $\mathrm{P}$.

\subsection{Analysis of lattice dimension}

In the present study, a two-dimensional square lattice $(310 \times 310)$ with periodic boundary condition has been implemented. Although such a boundary condition provides an infinite surface-like characteristic to the lattice, the number of nodes is kept fixed and finite. The nodes get filled with a pre-defined percentage of agents, according to the initial values. As the agents interact within the lattice, its dimensions can influence the final product concentration. It is necessary therefore to study the sensitivity of product formation to lattice dimension. This is done 


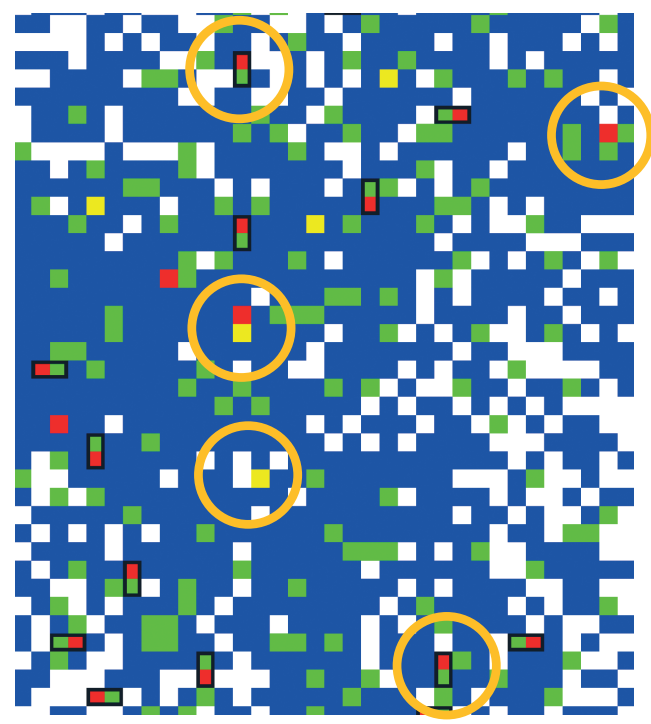

Figure 4. A snapshot of a simulation reaction performed after $X$ iterations for 57,660 water molecules (blue), 3844 substrate molecules (white), 961 enzyme (green) and inhibitor (yellow) molecules in a 96100cell domain with probability values (see table 2 ). The product molecules are colored in red.

by fixing the initial input parameter values, so that the following reaction equation can be followed:

$$
\mathrm{E}+\mathrm{S} \rightarrow \mathrm{ES} \rightarrow \mathrm{EP} \rightarrow \mathrm{E}+\mathrm{P}
$$

The parameters Join (WS), Join (WP), Join (EP), Join (EI), Join (ES), Break (ES), Break (WS), Break (WP), Break (EI), Break (ES) and Transition (EP to ES) are initialized to zero. To eliminate the stochastic fluctuations generated due to the probabilistic nature of the initial parameter values, the non-zero parameters of the model are initialized to 1 . This means that the probability of an event to occur is $100 \%$. Note that the relative concentrations of the agents are kept same for different lattice dimensions. To assess the model sensitivity, simulations are run for different lattice dimensions: $60 \times 60,110 \times 110,160 \times 160,210 \times 210,260 \times 260,310 \times 310,360 \times$ $360,410 \times 410$ and $460 \times 460$.

Figure 5 shows the variation of the final product $(\mathrm{P})$ concentration with lattice dimension. For each simulation run, the model parameters were kept constant to their standard values (see table 2) and the number of iterations was fixed at 3000 to determine the final product $\mathrm{P}$ concentration. Due to the absence of any reversible step, the entire substrate $\mathrm{S}$ gets converted to product $\mathrm{P}$. The lattice size was subsequently changed after each set to obtain a new $\mathrm{P}$ concentration. As seen in figure 5, the relative percentage conversion of $\mathrm{P}$ remains constant irrespective of the increase in lattice dimension. This is because increasing the lattice size and maintaining the relative percentage occupancy of nodes of the lattice will increase the number of interactions required to reach completion of the reaction linearly, since increasing the number of agents will require more reactions to be completed before reaching completion. Assuming extended von Neumann neighborhood for reaction, with each agent making the reaction complex bigger the reaction radius still stays at 2 units in four directions. Therefore an increase in lattice dimension does not automatically indicate an increase in product concentration, if the model parameter values and the lattice density are unchanged. However, the physical time corresponding to a discrete 


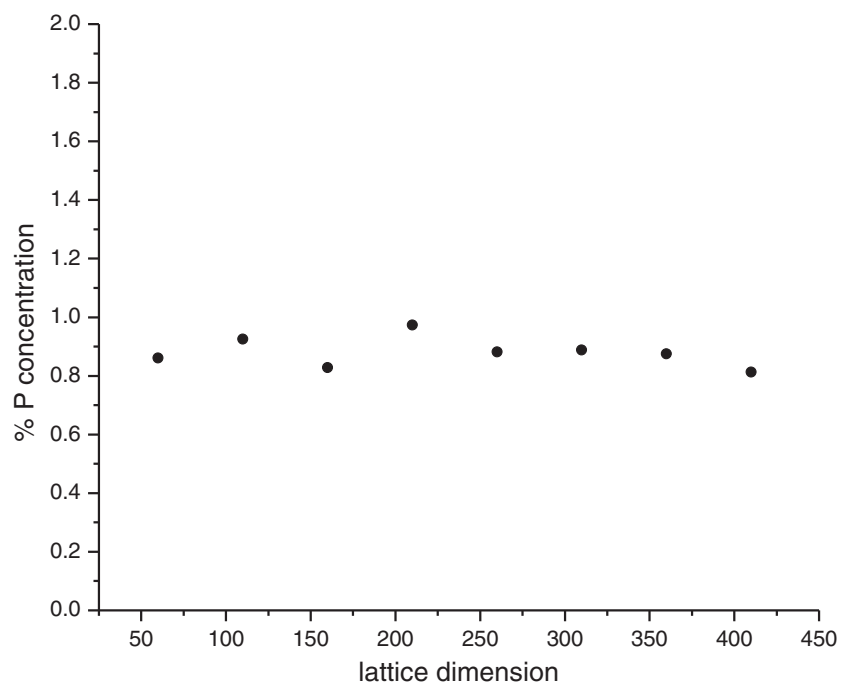

Figure 5. Variation of product concentration measured for each simulation vs. lattice dimension, after 3000 iterations.

time step will be lower in the case with a larger lattice dimension. But, as both cases describe the same process (and therefore the same total real physical reaction time), more time steps will be needed as the grid size increases. However, with computational time as an obvious limitation for these kinds of applications, a trade-off is accepted between time and reliability.

\subsection{Variability analysis of model parameters}

As mentioned earlier, the variation in product concentration on reaching the equilibrium obtained after 3000 iterations is studied and the final product concentration $(\mathrm{P})$ is considered for the model interpretation. It is important to note that the random seed value is kept constant, so that each set experiences a similar set of random values generated by the program during probabilistic rule application. Similar set of random values have been taken each time, so that the sensitivity analysis suffers less from stochastic fluctuations. Moreover, to increase the accuracy measure of the final attained product concentration for each set of initial parameter values, the average product concentration over 100 simulation sets is considered. As mentioned earlier, the step increment of the parameters is fixed at 0.01 . This value is assumed to be a compromise between computational time requirement and accuracy. The total time taken to simulate the model for 12 variables is approximately 22 days using the computational resources of the Flemish Supercomputer Centre (VSC).

While performing the simulations, the values of each of parameters except the one under consideration are fixed to their standard values. Figure $6(a-1)$ shows the variation of the product concentration with respect to the changing parameter, measured after 3000 iterations. It is essential to note that although the simulations are stochastic in nature, the different realizations cannot be shown in multiple details as they are essentially evolutions of a two-dimensional array. As such only the characteristics are shown, as indicated in these figures. However, since the variability is less than $1 \%$, the moments of the distribution such as mean and variance do not provide any extra information for these stochastic simulations and hence are not shown. An attempt has 

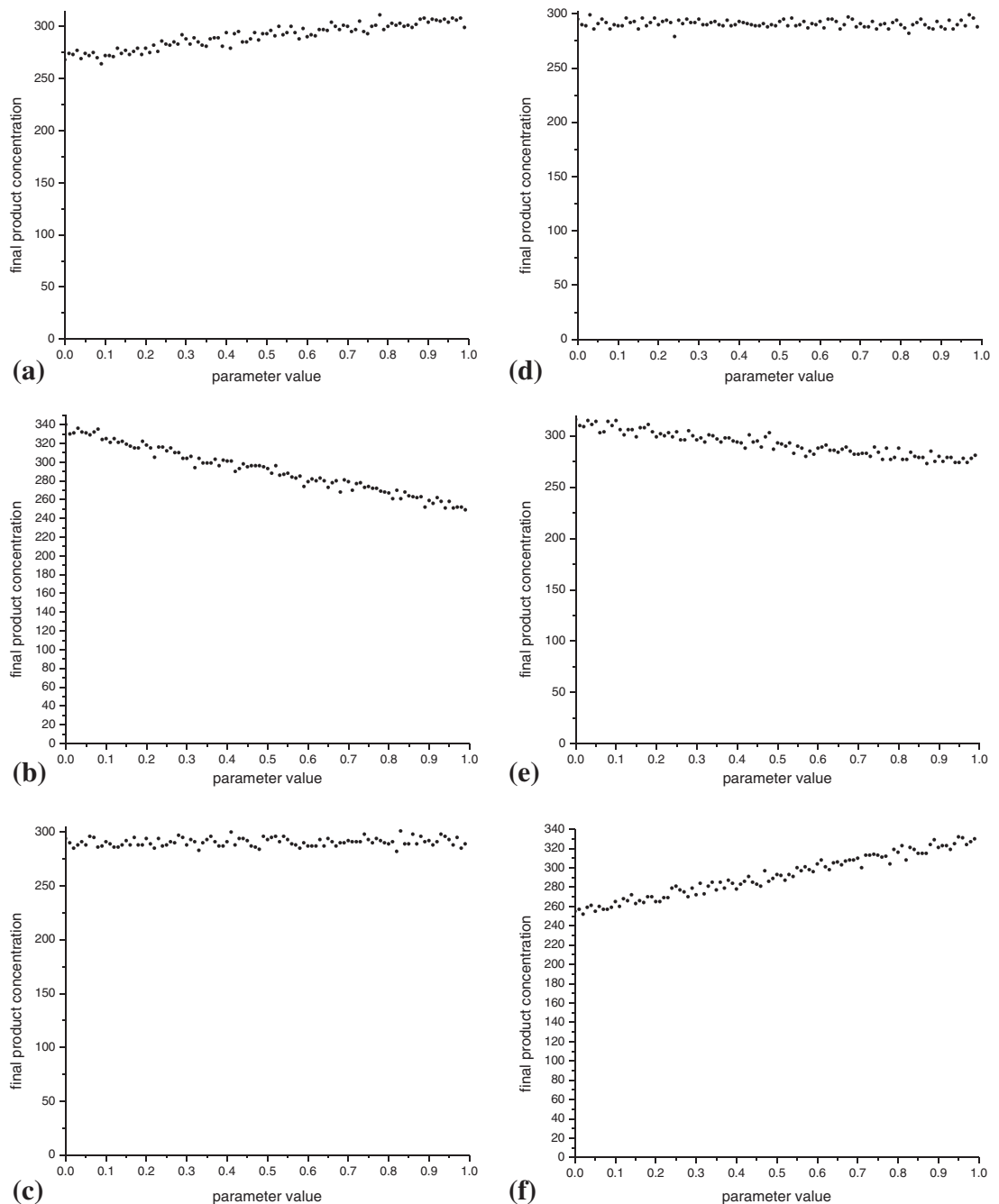

Figure 6. Variation of final product concentration P with (a) Join (ES); (b) Break (ES); (c) Join (EI); (d) Break (EI), the changing probability parameter has almost no effect on the final product concentration; (e) Join (EP); (f) Break (EP); (g) Join (WS), the product concentration decreases as the parameter value is increased; (h) Break (WS); (i) Join (WW), the parameter shows no significant fluctuation to the changing parameter value; (j) Break (WW); (k) Trans (ESEP); (l) Trans (EPES) probability parameter (lattice grid $=310 \times 310)$.

been made in this section to correlate these variations with the general kinetic properties and the enzymatic model. With increasing value of joining probability $\left(\mathrm{P}_{\mathrm{J}}\right)$ between $\mathrm{E}$ and $\mathrm{S}(\mathrm{Join}(\mathrm{ES})$ ), the tendency of $\mathrm{S}$ forming a complex with $\mathrm{E}$ increases thereby increasing the chance of formation of $\mathrm{P}$. Figure $6 \mathrm{a}$ shows an increasing trend in final product concentration as the probability value increases which satisfy the general perception of a forward kinetic reaction. It must be noted that even when Join (ES) is zero, $\mathrm{P}$ can still be formed because of $\mathrm{E}$ and $\mathrm{S}$ can be adjacent to each other due to free movement rule application. As the value of breaking probability $\left(\mathrm{P}_{\mathrm{B}}\right)$ between $\mathrm{E}$ and $\mathrm{S}$ (Break (ES)) increases, the chances of conversion from ES to EP complex are reduced. 

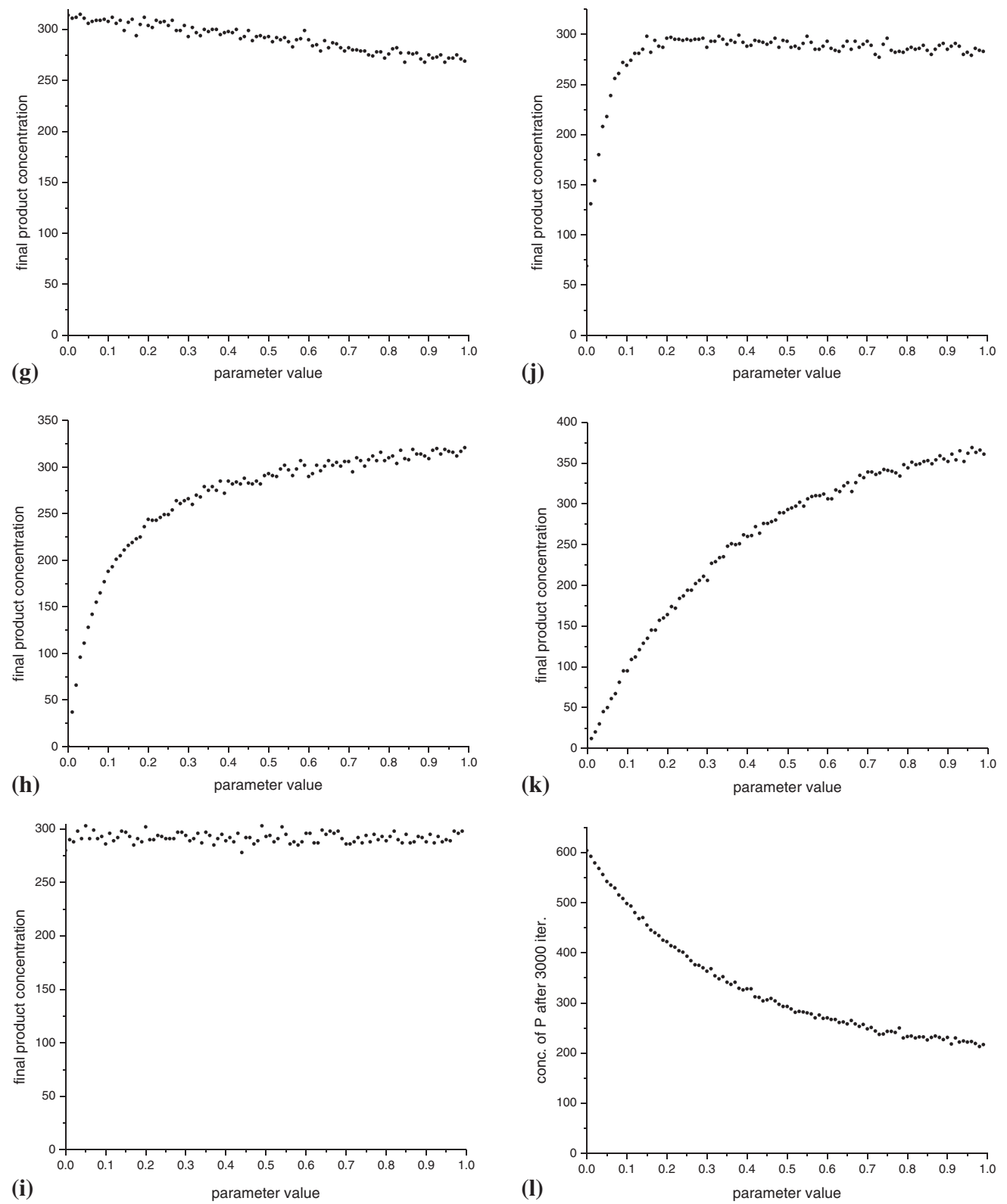

Figure 6. continued.

This decreasing trend can be seen in figure $6 \mathrm{~b}$. Variations for probability of joining and breaking between enzyme and inhibitor have almost no effect on the final product concentration at lower concentrations of the inhibitor. However, the effect of inhibition is visible when inhibitor concentration is comparable to the concentration of substrate and the inhibition activity can be carried forward to next iteration steps. To implement this, simulations have been performed in which the reaction structure for inhibitor has been modified to $\mathrm{E}+\mathrm{I} \rightleftarrows \mathrm{EI} \rightleftarrows \mathrm{XC} \rightleftarrows \mathrm{X}+\mathrm{C}$. Here 
$\mathrm{X}$ and $\mathrm{C}$ are considered as two pseudo fitting agents. The $\mathrm{XC}$ complex captures the inhibition activity as a memory in the reaction. The probability of transition from EI to $\mathrm{XC}$ is equivalent to CAJoinEI and the probability of transition from $\mathrm{XC}$ to $\mathrm{EI}$ is equivalent to CABreakEI. The other joining and breaking probabilities are kept equal to 1 so that they do not affect the application of other rules. The variability analysis for CAJoinEI and CABreakEI so performed with the increased substrate concentration shows expected variation. With increasing value of joining probability $\left(\mathrm{P}_{\mathrm{J}}\right)$ between $\mathrm{E}$ and I (Join (EI)), the tendency of $\mathrm{S}$ forming a complex with E reduces thereby decreasing the chance of formation of $\mathrm{P}$ (see figure $6 \mathrm{c}$ ). For increasing value of breaking probability $\left(\mathrm{P}_{\mathrm{B}}\right)$ between $\mathrm{E}$ and I (Break $(\mathrm{EI})$ ), free $\mathrm{E}$ increases which can react with $\mathrm{S}$ for ES complex formation, hence increase chance of formation of $P$ (see figure $6 \mathrm{~d}$ ). The joining probability between $\mathrm{E}$ and $\mathrm{P}$ (Join (EP)) represents the first step of reverse reaction and transformation from $\mathrm{P}$ to $\mathrm{S}$. If there is a higher joining probability, then the feasibility of the reverse reaction simultaneously increases by a certain margin. The decreasing concentration of $\mathrm{P}$ is shown in figure 6e with the increase in parameter value. Inversely, with increasing breaking probability of $\mathrm{P}$ from $\mathrm{E}$ (Break (EP)), the feasibility of the reverse reaction decreases and concentration of $P$ remains high (see figure $6 f$ ). With this, the availability of $E$ to interact with $S$ catalysing the reverse reaction increases. The joining probability between $\mathrm{W}$ and $\mathrm{S}$ (Join (WS)) represents the affinity of S towards W. This parameter is equivalent to the adsorption affinity between water and other agents as found in natural systems. With an increasing value of Join (WS), the resultant concentration of final $\mathrm{P}$ decreases (see figure $6 \mathrm{~g}$ ). The reverse explanation is applicable for the breaking probability between W and S (Break (WS)). With increasing Break (WS), there are more 'free' $S$ available to interact with $E$ and hence participate in the forward reaction for the formation of $\mathrm{P}$ (see figure 6h). But interestingly, the figure shows an exponentially increasing concentration which can be explained by the order of rule applications. The joining probability between $\mathrm{W}$ and $\mathrm{W}$ (Join (WW)) has no effect on the final P concentration (see figure 6i). As more $\mathrm{W}$ joins with $\mathrm{W}$, there is increasingly less free $\mathrm{W}$ available to interfere with $\mathrm{S}$ and hence free $\mathrm{S}$ can interact with $\mathrm{E}$ to participate in the forward reaction. For the breaking probability between W and W (Break (WW)), there is an initial steep increase and then gradually a decreasing behavior in $\mathrm{P}$ concentration, as shown in figure 6j. In a standard pseudo reaction system, substrate molecules are shown to have affinity towards water molecules. The same property is also observed in water-soluble compounds. Furthermore, physical observations show that water molecules do interact with each other due to their polar structure. It is observed that the simulation results for the effect of variation of joining and breaking probability between two water molecules are not much sensitive to the variation of the joining probability as it is to the variation of the breaking probability. The variability analysis performed in this study reveals that the two most sensitive parameters of the model presented in this study are the transition probability of ES to EP (Trans (ESEP)) and the transition probability of EP to ES (Trans (EPES)). These are the forward and reverse probabilistic rules controlling the transition step of the model. Increasing the transition probability (Trans (ESEP)) results in more EP complexes being formed due to forward reaction while increasing transition probability (Trans (EPES)) results in higher conversion of EP to ES, facilitating backward reaction, as shown in figure 6k and 61 respectively.

\section{Conclusions}

Sensitivity analysis of the parameters in the first order enzyme kinetic model confirms the effectiveness of the CA model to define and predict general chemical kinetics. From the model, kinetic concentrations were obtained which matched the general observations. The parameter variability 
analysis on $\mathrm{P}_{\mathrm{J}}, \mathrm{P}_{\mathrm{B}}$ and $\mathrm{P}_{\mathrm{T}}$ shows Join(ES), Join(EP), Break(ES), Break(EP), Trans(ESEP), Trans(EPES) to have higher sensitivity and Join(EI), Join(WW), Break(EI), Break(WW) to have lesser sensitivity on the model output product concentration. The underlying cause of this behavior is the various inter-relation effects of the different probabilistic input parameter values used in the CA model. The present study allows understanding the sensitivity of the cellular automata lattice dimension and the probabilistic model parameters. The knowledge gained from this study will be further used to implement the stochastic model for specific biochemical reactions. It is, however, important to note that this model is a simplified formulation and does not represent any enzymatic reaction in particular. It is therefore necessary to calibrate the model to represent specific biochemical reactions, especially using the effect of molecular memory to validate the model. This would be helpful to standardize the model parameters thereby represent specific biochemical reactions, which can substantiate experimental results.

\section{Acknowledgement}

Pieter Van der Weeën, KERMIT Knowledge-based Systems, Ghent University is acknowledged for fruitful discussions related to the model. The infrastructure of the Flemish Supercomputer Centre (VSC) is financed by the Hercules Foundation and the Flemish Government department EWI.

\section{References}

Apte A, Cain J W, Bonchev D G and Fong S S 2008 Cellular automata simulation of topological effects on the dynamics of feed-forward motifs. J. Biol. Eng. 2(2): 1-12

De Levie R 2000 Stochastics, the basis of chemical dynamics. J. Chem. Education 77(6): 771-774

Deutsch A and Dormann S 2005 Cellular automaton modelling of biological pattern formation. Boston: Birkhauser

Goutsias J 2007 Classical versus stochastic kinetics modeling of biochemical reaction systems. Biophys. J. 92(7): 2350-2365

Kar S, Ganai S, Dutta A, Dutta D and Chaudhuri S 2010 A sensitivity analysis study of enzyme inhibition kinetics through cellular automata. In: AIP Conference Proceedings (ICMOC 2010) 1298, 1, 301-306

Kar S, Nag K, Dutta A, Constales D and Pal T 2014 An improved Cellular Automata model of enzyme kinetics based on Genetic Algorithm. Chem. Eng. Sci. 110: 105-118

Kier L B, Cheng C K and Testa B 1999 Cellular automata models of biochemical phenomena. Future Generation Comput. Syst. 16(2-3): 273-289

Kier L B, Seybold P G and Cheng C K 2005 Cellular automata modeling of chemical systems. A textbook and laboratory manual. Netherlands: Springer

Martin O, Odlyzko A and Wolfram S 1984 Algebraic properties of cellular automata. Commun. Math. Phys. 93(2): 219-258

Seybold P G, Kier L B and Cheng C K 1997 Simulation of first-order chemical kinetics using cellular automata. J. Chem. Inf. Comput. Sci. 37: 386-391

Van der Weeën P, Baetens J M and De Baets B 2011 Design and parameterization of a stochastic cellular automaton describing a chemical reaction. J. Comput. Chem. 32(9): 1952-1961

Von Neumann J 1966 Theory of self-reproducing automata. Burks A W (ed.) Urbana: University of Illinois Press

Weimar J R 2002 Cellular automata approaches to enzymatic reaction networks. $5^{\text {th }}$ International Conference on Cellular Automata for Research and Industry ACRI 2002. Bandini S, Chopard B, Tomassini M (eds.), Springer Lecture Notes in Computer Science 2493, 294-303

Wolfram S 1984 Cellular automata as models of complexity. Nature 311: 419-424 